\title{
Chemical composition and Biological studies of Ficus benjamina
}

\author{
Muhammad Imran ${ }^{1}$, Nasir Rasool', Komal Rizwan ${ }^{1}$, Muhammad Zubair ${ }^{1}$, Muhammad Riaz', \\ Muhammad Zia-Ul-Haq ${ }^{2}$, Usman Ali Rana ${ }^{3}$, Ayman Nafady ${ }^{4}$ and Hawa ZE Jaafar 5,6*
}

\begin{abstract}
Background: Current study has been designed to estimate the possible antioxidant, antimicrobial and hemolytic potential of Ficus benjamina different parts (leaves, stem and root).

Results: All examined extracts and fractions were significantly rich in antioxidants and exhibited potent antimicrobial activity. GC/MS analysis of essential oil identified four compounds in stem and eight compounds in root, respectively. HPLC analysis indicated four phenolic compounds (chlorogenic, $p$-coumaric, ferulic and syringic acids) in roots, three (chlorogenic $p$-coumaric and ferulic acids) in stem and only one (caffeic acid) in leaves. Extracts of all three parts of $F$. benjamina exhibited substantial hemolytic activity.
\end{abstract}

Conclusions: Considering these results, it is concluded that $F$. benjamina can be used as a potential source for the exploration of new antioxidant compounds and antimicrobial agents.

Keywords: Ficus benjamina, Phenolic acids, HPLC analysis, Cytotoxicity, Antioxidant activity

\section{Background}

Ficus benjamina L. (Moraceae), locally known as weeping fig, is a multipurpose tree found in various parts of Pakistan. F. benjamina is native to a large area including India, southern China, Southeast Asia, Malaysia, the Philippines, northern Australia, and the islands of the South Pacific [1]. F. benjamina is cultivated in many parts of the world including American Samoa (Tutuila), French Polynesia (cult.), Marshall Islands (Kwajalein (cult.), Majuro (cult.), Tonga as well as Florida, in the United State [2]. It grows as a large evergreen shrub, up to $8 \mathrm{~m}$ tall, with nearly $10 \mathrm{~m}$ wide spreading crown and drooping shoots with young slender twigs. The plant is well known due to its medicinal potential. Its latex and some fruit extracts are used by indigenous communities to treat skin disorders, inflammation, piles, vomiting, leprosy, malaria, nose-diseases and cancer besides the use as a general tonic. The plant is also used as antimicrobial, antinociceptive, antipyretic, hypotensive and anti-dysentery remedy.

\footnotetext{
*Correspondence: hawazej@gmail.com

${ }^{5}$ Department of Crop Science, Faculty of Agriculture, 43400 UPM Serdang,

Selangor, Malaysia

${ }^{6}$ University Community Transformation Centre, Industry-Community Engagement, 43400 UPM Serdang, Selangor, Malaysia

Full list of author information is available at the end of the article
}

The leaves and twigs are used as insect repellant [3-6]. The leaves, bark and fruits of $F$. benjamina contain various bioactive constituents like cinnamic acid, lactose, naringenin, quercetin, caffeic acid and stigmasterol [5].

Despite its wide use, some literature is available about the chemistry and the biological properties of this plant [7-10]. In this context, as part of our studies on indigenous flora of Pakistan [11-21], the present study was conducted to evaluate some chemical and biological characteristics of F. benjamina.

\section{Results and discussion}

Percent yield of extracts and fractions

Determination of extract yield is an important indicator and first step during evaluation of antioxidant capacity of extracts of plants. The percent yield of different extracts and fractions (methanol extract, $n$-hexane, chloroform, ethyl acetate, $n$-butanol) of stem, root and leaves was found to be in the range of $9.7-18 \mathrm{~g} / 100 \mathrm{~g}$, $8-20 \mathrm{~g} / 100 \mathrm{~g}$ and $8.3-23.34 \mathrm{~g} / 100 \mathrm{~g}$, respectively. The maximum yield was exhibited by methanol extract of leaves $(23.34 \mathrm{~g} / 100 \mathrm{~g})$. The methanol extracts of root $(20 \mathrm{~g} / 100 \mathrm{~g})$ and stem $(18 \mathrm{~g} / 100 \mathrm{~g})$ also showed good yield. Our results of maximum methanolic extract yield for $F$. benjamina leaves agree with those previously
(C) Chemistry Central

(c) 2014 Imran et al.; licensee Chemistry Central Ltd. This is an open access article distributed under the terms of the Creative Commons Attribution License (http://creativecommons.org/licenses/by/2.0), which permits unrestricted use, distribution, and reproduction in any medium, provided the original work is properly cited. 
reported Ficus specie leaves sample (18.12\%) [22]. It is believed that percentage yield of extracts depends on various factors, like plant part, season and maturity of plant part, and agroclimatic conditions from where that part is collected.

\section{Total phenolic contents (TPC) and total flavonoid contents (TFC)}

The extract and fractions of stem, root and leaves exhibited TPC values as was measured by Folin-Ciocalteu reagent procedure in the range of 50.80-735.11, 55.02-705.11, 52.61-617.50 (Gallic acid equivalent, (GAE) $\mathrm{mg} / 100 \mathrm{~g}$ ) of dry extract, respectively. The methanol extract and $n$-butanol fractions showed greater amount of phenolic contents. The $n$-hexane fractions of all three parts exposed minimum phenolic contents with a maximum TPC value in leaves (12.61 GAE mg/100 g). The chloroform and ethyl acetate fractions indicated considerable phenolic contents, showing maximum value 263.85 (ethyl acetate) for stem (Table 1 ). The TPC values for methanol extracts were found to be in the range of 531-573.06 GAE mg/100 g. The $n$-butanolic fractions showed higher phenolic contents as 735.11 (GAE $\mathrm{mg} / 100 \mathrm{~g}$ ) and showed maximum values out of all fractions of three parts. The $n$-hexane fractions of all three parts showed the lowest phenolic contents. The chloroform and ethyl acetate fractions indicated considerable phenolic contents, showing maximum value 263.85 (ethyl acetate) for stem. Our results agree with earlier studies [22] that report TPC values of 100 GAE mg/100 g.

The range of TFC for stem was 60.46-1466.3, for root 112.38-1554.02 and for leaves 110.45-1492.1 CE mg/100 g of dry extract, respectively. The methanol extract (1554.02) and $n$-butanolic fraction (1545.4) of root showed maximum values of flavonoid contents. The $n$-hexane fractions exhibited minimum quantity of flavonoids out of all fractions and its maximum value was 112.38. Ethyl acetate and chloroform fractions also revealed substantial values of TFC contents (Table 1). The root methanol extract and $n$-butanolic fraction showed maximum values of flavonoid contents (1554.02 and 1545.4, respectively). The $n$-hexane fractions showed lower flavonoid contents as compared to other extract and fractions. Ethyl acetate and chloroform fractions also revealed substantial values of TFC, showing maximum amount of flavonoids. However, the methanol extract of root showed maximum contents of TFC (1554.02). In addition, its $n$-butanolic fraction also disclosed tangible value (1592.1). Hence, root contained higher amounts of flavonoids than stem and leaves.

Table 1 Total phenolic content, total flavonoid content and antioxidant activity of different parts of $F$. benjamina

\begin{tabular}{|c|c|c|c|c|}
\hline \multirow[t]{2}{*}{ Antioxidant assay } & \multicolumn{4}{|c|}{ Plant parts } \\
\hline & Crude extracts and fractions & Stem & Root & Leaves \\
\hline \multirow[t]{5}{*}{ Total phenolic contents (GAE mg/100 g) } & Methanol & $539.17 \pm 3.21^{\mathrm{e}}$ & $573.06 \pm 2.74^{d}$ & $531.76 \pm 4.90^{\mathrm{e}}$ \\
\hline & n-butanol & $735.11 \pm 3.42^{\mathrm{a}}$ & $705.48 \pm 3.42^{b}$ & $650.17 \pm 5.22^{c}$ \\
\hline & Chloroform & $90.28 \pm 0.11^{j}$ & $122.87 \pm 0.65^{i}$ & $66.76 \pm 1.07^{\mathrm{k}}$ \\
\hline & Ethyl acetate & $263.85 \pm 1.71^{f}$ & $237.19 \pm 1.71^{9}$ & $157.19 \pm 1.71^{\mathrm{h}}$ \\
\hline & n-hexane & $7.80 \pm 0.211^{k}$ & $10.02 \pm 0.211^{i}$ & $12.61 \pm 0.211^{\prime}$ \\
\hline \multirow[t]{5}{*}{ Total flavonoid contents (CE mg/100 g) } & Methanol & $724.60 \pm 0.89^{f}$ & $1654.00 \pm 8.93^{b}$ & $1592.20 \pm 8.93^{c}$ \\
\hline & n-butanol & $1566.40 \pm 9.52^{d}$ & $1665.30 \pm 9.52^{\mathrm{b}}$ & $1780.80 \pm 9.52^{\mathrm{a}}$ \\
\hline & Chloroform & $103.20 \pm 2.68^{k}$ & $491.20 \pm 4.55^{\mathrm{h}}$ & $262.70 \pm 2.98^{j}$ \\
\hline & Ethyl acetate & $329.60 \pm 4.76^{i}$ & $807.90 \pm 4.76^{\mathrm{e}}$ & $552.20 \pm 4.76^{9}$ \\
\hline & n-hexane & $4.50 \pm\left. 0.25\right|^{j}$ & $6.40 \pm 0.12 \mathrm{j}^{j}$ & $8.40 \pm 0.12^{j}$ \\
\hline \multirow[t]{5}{*}{$\mathrm{DPPH}, \mathrm{IC} \mathrm{C}_{50}(\mu \mathrm{g} / \mathrm{ml})$} & Methanol & $50.10 \pm 3.23^{i}$ & $58.81 \pm 4.50^{i}$ & $49.86 \pm 3.39^{i}$ \\
\hline & n-butanol & $152.35 \pm 4.10^{9}$ & $158.44 \pm 3.29^{9}$ & $147.46 \pm 3.85^{9}$ \\
\hline & Chloroform & $176.04 \pm 4.54^{f}$ & $221.22 \pm 4.20^{\mathrm{e}}$ & $103.96 \pm 2.06^{h}$ \\
\hline & Ethyl acetate & $228.79 \pm 5.27^{\mathrm{de}}$ & $237.1 \pm 4.69^{d}$ & $223.00 \pm 2.68^{e}$ \\
\hline & $n$-hexane & $554.13 \pm 7.39^{b}$ & $580.75 \pm 5.89^{a}$ & $436.21 \pm 7.27^{c}$ \\
\hline \multirow[t]{5}{*}{$\%$ Inhibition in linoleic acid system } & Methanol & $78.16 \pm 3.41^{\mathrm{b}}$ & $85.87 \pm 3.59^{\mathrm{a}}$ & $69.81 \pm 3.00^{c}$ \\
\hline & n-butanol & $49.66 \pm 2.98^{\mathrm{ef}}$ & $81.48 \pm 3.59^{\mathrm{ab}}$ & $50.48 \pm 2.47^{\mathrm{e}}$ \\
\hline & Chloroform & $16.94 \pm 1.44^{j}$ & $58.52 \pm 2.94^{d}$ & $42.41 \pm 2.36^{\mathrm{fg}}$ \\
\hline & Ethyl acetate & $34.79 \pm 2.41^{h}$ & $24.50 \pm 1.88^{i}$ & $37.95 \pm 1.78^{g h}$ \\
\hline & n-hexane & $35.65 \pm 2.00^{g h}$ & $20.57 \pm 0.63^{i j}$ & $26.82 \pm 1.85^{i}$ \\
\hline
\end{tabular}




\section{Antioxidant activity}

Free radicals are the major contributors to several clinical disorders such as diabetes mellitus, cancer, renal failures and degenerative diseases as they disturb natural defence mechanisms. These disorders can be prevented by supplementing the body's natural antioxidant defence. Plant extracts potentiate human antioxidant defence system and are antioxidant of choice because of their lower toxicity and side effects over the synthetic ones.

Methanol extracts of stem, root and leaves exhibited $\mathrm{IC}_{50}$ values of $50.1,58.81$ and $49.86 \mu \mathrm{g} / \mathrm{ml}$, respectively. The maximum value of $\mathrm{IC}_{50}$ was demonstrated by root's fraction of $n$-hexane $(580.75 \mu \mathrm{g} / \mathrm{ml})$, indicating that this fraction showed minimum free radical scavenging activity. Unlike $n$-hexane fraction, chloroform and ethyl acetate fractions exhibited lower values of $\mathrm{IC}_{50}$ as showed in Table 1 . The methanol extract and $n$-butanolic fractions showed maximum free radical scavenging activity. The $n$-hexane fractions of root revealed maximum value of $\mathrm{IC}_{50}(580.75 \mu \mathrm{g} / \mathrm{ml})$. Methanol and $n$-butanol fractions exhibited the lowest $\mathrm{IC}_{50}$ values, showing a maximum value $(158.34 \mu \mathrm{g} / \mathrm{ml})$ for root.

The methanol extract and $n$-butanol fraction showed greater percent inhibition of linoleic acid system, compared to other fractions. The percent inhibition in linoleic acid system for stem wass in the range of $16.94-78.16 \%$, in root $20.57-85.87 \%$ and in leaves $26.82-69.81 \%$. The maximum percent inhibition was determined by methanol extract (85.87) and butanol fraction (81.48) of root. The results of these experiments revealed that antioxidant potential of plant increased linearly with the increase in concentration. The methanol extract as well as fractions of root exhibited a linear rise in absorbance value for various concentrations $0.56 \mathrm{~nm}: 2.5(\mathrm{mg} / \mathrm{ml}), 0.87 \mathrm{~nm}: 5(\mathrm{mg} / \mathrm{ml})$, $1.03 \mathrm{~nm}: 7.5(\mathrm{mg} / \mathrm{ml})$ and $1.49 \mathrm{~nm}: 10(\mathrm{mg} / \mathrm{ml})$ (Table 2). The presence of phenolic compounds might be the reason for reducing power. Literature reports [23-25] indicate that the reducing power of bioactive compounds is associated with antioxidant activity. The results of this assay indicated that the plant is a good source of antioxidants with high reducing power.

\section{Antimicrobial activity}

The extracts and fractions of stem, root and leaves exhibited considerable antimicrobial activity against four bacterial and two fungal strains (Table 3). The range of antimicrobial activity expressed as diameters of inhibition zone (DIZ) for stem was $10.5 \mathrm{~mm}$ ( $n$-hexane) - $22.83 \mathrm{~mm}$ ( $n$-butanol). All the butanol fractions exhibited strong activity. Methanol extract $(22.63 \mathrm{~mm}$ against $P$. aeruginosa) and $n$-butanolic fraction (22.83 against B. subtilis) of stem showed substantial activity. The $n$-hexane, chloroform and ethyl acetate sprouted moderate value of DIZ, with maximum value disclosed by ethyl acetate $(16.88 \mathrm{~mm})$. The stem extract and fractions revealed the following order of antimicrobial potential against $B$. cerus; methanolic $>n$-butanolic $>$ ethyl acetate $>$ chloroform $>n$-hexane.

Stem extract and fractions exhibited a different order against the two fungal strains tested. Root extract and fractions disclosed DIZ values in the range of $12.96 \mathrm{~mm}$ (n-hexane) to $17.73 \mathrm{~mm}$ ( $n$-butanol) against bacterial strains. The maximum value of DIZ was exhibited by methanol extract and $n$-butanolic fraction. The chloroform and ethyl acetate fractions of root also showed good values. Methanol, chloroform, ethyl acetate and $n$-butanolic fractions of root exhibited moderate activity with maximum activity given by $n$-butanolic fraction against $B$. cereus. Overall, $n$-hexane faction of root showed poor activity.

In case of fungal strains, chloroform and ethyl acetate fractions of root evinced higher antimicrobial potential as compared to methanol and $n$-butanolic fractions. The root extract and fractions of methanol, $n$-butanol, chloroform, ethyl acetate and $n$-hexane exhibited the following values of DIZ against A. niger: 14.60, 14.98, $17.15,16.38,14.70 \mathrm{~mm}$, respectively. The butanol fraction from leaves again showed higher antimicrobial potential as is evident from its values $19.50 \mathrm{~mm}$ and $19.75 \mathrm{~mm}$ against $B$. cerus and C. albicans, respectively. The methanol, $n$-hexane, chloroform and ethyl acetate fractions of leaves showed moderate activity with considerable value shown by chloroform fraction $(12 \mathrm{~mm})$. However, $n$-butanol fraction of leaves showed strong activity against $B$. cerus. Comparatively, $n$-hexane and ethyl acetate fractions denoted lower antimicrobial potential. Results of present study indicate that $F$. benjamina extracts can be used as a potential antimicrobial agent to inhibit the growth of various dangerous microbes.

The results of minimum inhibitory concentration (MIC) are presented in Table 4. The MIC values were inverse to antimicrobial values; the butanolic fraction of stem showed the maximum antimicrobial. The range of its MIC values was found to be $0.65-1.79 \mathrm{mg} / \mathrm{ml}$, indicating that it might act as an antimicrobial at low concentrations. Similarly, $n$-butanolic fractions of root and leaves exhibited maximum antimicrobial activity with MIC of $0.77-1.99 \mathrm{mg} / \mathrm{ml}$ for root and $0.7-1.5 \mathrm{mg} / \mathrm{ml}$ for leaves, respectively. Similar results were showed by the MIC analyses of fungal strains (Table 4).

\section{HPLC analysis of phenolic acids}

The HPLC analysis for the presence of phenolic acids permitted the identification of 5 phenolic acids, three in stem, four in root and one in leaves. Results are presented in Table 5. Sirisha and co-workers [5] reported the presence of ursolic, $\alpha$-hydroxy ursolic, protocatechuic and maslinic acids in Ficus species, while cinnamic 
Table 2 Reducing potential of different parts of $\boldsymbol{F}$. benjamina

\begin{tabular}{|c|c|c|c|c|}
\hline \multirow{2}{*}{$\begin{array}{l}\text { Plant extracts } \\
\text { and fractions }\end{array}$} & \multirow{2}{*}{$\begin{array}{l}\text { Concentration } \\
(\mathrm{mg} / \mathrm{ml})\end{array}$} & \multicolumn{3}{|c|}{ Plant parts } \\
\hline & & Stem & Root & Leaves \\
\hline \multirow[t]{4}{*}{ Methanol } & 2.5 & $0.64 \pm 0.003^{\mathrm{rs}}$ & $0.56 \pm 0.003^{\mathrm{uv}}$ & $0.39 \pm 0.00^{z}$ \\
\hline & 5.0 & $1.22 \pm 0.015^{e}$ & $0.87 \pm 0.001^{\mathrm{klm}}$ & $0.57 \pm 0.001^{\text {tuv }}$ \\
\hline & 7.5 & $1.39 \pm 0.006^{c}$ & $1.03 \pm 0.001^{9}$ & $0.79 \pm 0.001^{\text {no }}$ \\
\hline & 10.0 & $1.47 \pm 0.006^{\mathrm{ab}}$ & $1.49 \pm 0.001^{\mathrm{a}}$ & $1.41 \pm 0.003^{b c}$ \\
\hline \multirow[t]{4}{*}{$n$-butanol } & 2.5 & $1.02 \pm 0.006^{9}$ & $0.76 \pm 0.006^{\circ p}$ & $0.38 \pm 0.001^{z}$ \\
\hline & 5.0 & $1.10 \pm 0.006^{f}$ & $0.83 \pm 0.001^{I m n}$ & $1.18 \pm 0.006^{e}$ \\
\hline & 7.5 & $1.22 \pm 0.006^{\mathrm{e}}$ & $0.84 \pm 0.005^{1 \mathrm{mn}}$ & $1.28 \pm 0.029^{d}$ \\
\hline & 10.0 & $1.39 \pm 0.026^{c}$ & $0.91 \pm 0.001^{1 \mathrm{jk}}$ & $1.47 \pm 0.005^{\mathrm{ab}}$ \\
\hline \multirow[t]{4}{*}{ Chloroform } & 2.5 & $0.37 \pm 0.001^{z}$ & $0.37 \pm 0.001^{z}$ & $0.64 \pm 0.001^{\mathrm{rs}}$ \\
\hline & 5.0 & $0.39 \pm 0.001^{z}$ & $0.61 \pm 0.001^{\mathrm{stu}}$ & $0.93 \pm 0.001^{\mathrm{ij}}$ \\
\hline & 7.5 & $0.46 \pm 0.001^{x y}$ & $0.62 \pm 0.001 \mathrm{st}$ & $0.94 \pm 0.001^{i j}$ \\
\hline & 10.0 & $0.47 \pm 0.001^{x}$ & $0.91 \pm 0.001^{\mathrm{ijk}}$ & $0.96 \pm 0.001^{h i}$ \\
\hline \multirow[t]{4}{*}{ Ethyl acetate } & 2.5 & $0.48 \pm 0.001^{x}$ & $0.60 \pm 0.001^{\text {stu }}$ & $0.50 \pm 0.001^{w x}$ \\
\hline & 5.0 & $0.57 \pm 0.001^{\text {tuv }}$ & $0.88 \pm 0.001^{\mathrm{jkl}}$ & $0.59 \pm 0.001^{\mathrm{sv}}$ \\
\hline & 7.5 & $0.73 \pm 0.001^{p q}$ & $1.01 \pm 0.006^{g h}$ & $0.61 \pm 0.001^{\text {stu }}$ \\
\hline & 10.0 & $0.91 \pm 0.001^{\mathrm{ijk}}$ & $1.29 \pm 0.023^{d}$ & $0.64 \pm 0.001^{\mathrm{rs}}$ \\
\hline \multirow[t]{4}{*}{ n-hexane } & 2.5 & $0.48 \pm 0.001^{x}$ & $0.35 \pm 0.001^{z}$ & $0.68 \pm 0.001^{\mathrm{qr}}$ \\
\hline & 5.0 & $0.49 \pm 0.001^{w x}$ & $0.37 \pm 0.001^{z}$ & $0.79 \pm 0.003^{\text {no }}$ \\
\hline & 7.5 & $0.54 \pm 0.001^{v w}$ & $0.40 \pm 0.001^{y z}$ & $0.69 \pm 0.151^{q r}$ \\
\hline & 10.0 & $0.59 \pm 0.001^{\mathrm{sv}}$ & $0.81 \pm 0.001^{\mathrm{mno}}$ & $1.12 \pm 0.015^{f}$ \\
\hline
\end{tabular}

Values are means \pm SD of three separate experiments $(p<0.05)$. Different letters in superscript indicate significant differences within solvents and among various parts.

and caffeic acids and quercetin have been reported in leaves, bark and fruits of F. benjamina [6]. All the detected phenolic acids are known to have antimicrobial and antioxidant properties [26-28]. So these phenolic acids may be responsible for antimicrobial and antioxidant activities of Ficus benjamina.

\section{Evaluation of hemolytic activity}

Table 6 reports the hemolytic activity of different extracts and fractions from $F$. benjamina. The maximum hemolytic activity was shown by chloroform fractions of stem $(3.36 \%)$ and leaves (3.29\%). The mechanical stability of the erythrocytic membrane is a good indicator of cytotoxity. The percentage lysis of human erythrocytes was below $5.0 \%$ for all samples, so it can be expected that the extract and fractions have a no cytotoxity $[29,30]$.

\section{GC-MS analysis}

Nine chemical constituents have been identified from essential oil of stem and root of $F$. benjamina obtained by hydrodistillation and subsequently subjected to GC-MS analysis. Stem essential oil contained four compounds: 2-pentanone, hexadecanoic acid, palmitic acid, 9,12-octadecadienoic acid; roots contained eight compounds: methanamine, cyclopentanone, methyl-2 phenylindole, cyclopropaneoctanal, arsenous acid, hexadecanoic acid, palmitic acid and 9,12-octadecadienoic acid (Table 7).

\section{Materials and methods \\ Collection of plant material}

The stem, root and leaves of $F$. benjamina were collected from the Botanical Garden, University of Agriculture, Faisalabad, Pakistan and were further authenticated by the Taxonomist, Dr. Mansoor Hameed, Department of Botany, University of Agriculture, Faisalabad, Pakistan where a voucher specimen has been deposited.

\section{Preparation of extracts and fractions}

The stem, root and leaves of $F$. benjamina were washed with cold water to remove dust and other extraneous matter. The shaded dried parts of plant were grinded into powder form $(500 \mathrm{~g})$ and extracted with methanol at room temperature. The methanol extracts were concentrated with rotary evaporator. Then the methanol extracts of stem $(80 \mathrm{~g})$, root $(80 \mathrm{~g})$ and leaves $(80 \mathrm{~g})$ were further fractioned with different polarity based solvents. The stem methanolic extract was suspended in distilled water and fractioned with $n$-hexane $(10 \mathrm{~g})$, chloroform (8 g), ethyl acetate $(9.7 \mathrm{~g})$ and $n$-butanol (12 g). Root 
Table 3 Antimicrobial analyses of $\boldsymbol{F}$. benjamina against bacterial and fungal strains by disc diffusion assay

\begin{tabular}{|c|c|c|c|c|}
\hline \multirow[t]{2}{*}{ Microbial strains } & \multirow{2}{*}{$\begin{array}{l}\text { Plant extracts } \\
\text { and fractions }\end{array}$} & \multicolumn{3}{|c|}{ Diameter of inhibition Zone in $\mathrm{mm}$} \\
\hline & & Stem & Root & Leaves \\
\hline \multirow[t]{6}{*}{ Bacillus cerus } & Methanol & $22.5 \pm 0.87^{b}$ & $16.88 \pm 0.36^{\mathrm{ef}}$ & $12.00 \pm 0.29^{g h}$ \\
\hline & n-butanol & $22.25 \pm 0.72^{b}$ & $17.73 \pm 0.16^{\mathrm{e}}$ & $19.50 \pm 0.29^{c d}$ \\
\hline & Chloroform & $12 \pm 0.58^{\mathrm{gh}}$ & $15.63 \pm 0.22^{f}$ & $10.75 \pm 0.14^{\mathrm{hi}}$ \\
\hline & Ethyl acetate & $17 \pm 0.58^{e f}$ & $16.88 \pm 0.07^{\mathrm{ef}}$ & $10.00 \pm 0.58^{i}$ \\
\hline & n-hexane & $11.25 \pm 0.72^{\mathrm{hi}}$ & $12.99 \pm 0.02^{9}$ & $6.50 \pm 0.29^{j}$ \\
\hline & Ampiciline & $24.6 \pm 0.66^{\mathrm{a}}$ & $18.25 \pm 0.43 d^{e}$ & $20.75 \pm 0.72^{c}$ \\
\hline \multirow[t]{6}{*}{ Pseudomonas aerugonisa } & Methanol & $23.5 \pm 0.29^{a}$ & $16.75 \pm 0.43 d^{e}$ & $12.04 \pm 0.31^{\mathrm{fg}}$ \\
\hline & n-butanol & $22 \pm 0.87^{b}$ & $17.23 \pm 0.13^{d}$ & $19.13 \pm 0.07^{c}$ \\
\hline & Chloroform & $12.25 \pm 0.72^{\mathrm{fg}}$ & $15.88 \pm 0.36^{\mathrm{e}}$ & $10.5 \pm 0.29^{h}$ \\
\hline & Ethyl acetate & $16.75 \pm 0.72^{\text {de }}$ & $17.13 \pm 0.22 d^{e}$ & $10.25 \pm 0.43^{h}$ \\
\hline & n-hexane & $11 \pm 0.58^{g h}$ & $13.09 \pm 0.10^{f}$ & $7.50 \pm 0.29^{i}$ \\
\hline & Ampiciline & $24.38 \pm 0.79^{a}$ & $17.75 \pm 0.14^{d}$ & $20.25 \pm 0.43^{c}$ \\
\hline \multirow[t]{6}{*}{ Escherichia coli } & Methanol & $22.63 \pm 0.22^{b}$ & $16.47 \pm 0.59^{e f}$ & $11.75 \pm 0.14^{h}$ \\
\hline & n-butanol & $22.75 \pm 0.43^{b}$ & $16.23 \pm 0.71^{\mathrm{ef}}$ & $18.25 \pm 0.43^{c}$ \\
\hline & Chloroform & $11.57 \pm 0.33^{h}$ & $15.63 \pm 0.22^{f}$ & $11.58 \pm 0.33^{h}$ \\
\hline & Ethyl acetate & $16.88 \pm 0.65^{\text {de }}$ & $16.88 \pm 0.07^{\mathrm{de}}$ & $10.00 \pm 0.58^{i}$ \\
\hline & n-hexane & $10.61 \pm 0.35^{h i}$ & $13.34 \pm 0.24^{9}$ & $6.50 \pm 0.29^{j}$ \\
\hline & Ampiciline & $24.65 \pm 0.64^{a}$ & $17.87 \pm 0.71^{\mathrm{ef}}$ & $18.75 \pm 0.43^{c}$ \\
\hline \multirow[t]{6}{*}{ Bacillus subtilis } & Methanol & $22.5 \pm 0.29^{b}$ & $16.43 \pm 0.62^{e}$ & $12.08 \pm 0.29^{f}$ \\
\hline & n-butanol & $22.83 \pm 0.39^{b}$ & $16.34 \pm 0.64^{e}$ & $18.50 \pm 0.29^{c}$ \\
\hline & Chloroform & $12 \pm 0.58^{f}$ & $15.75 \pm 0.29^{e}$ & $12.13 \pm 0.65^{f}$ \\
\hline & Ethyl acetate & $16.63 \pm 0.79^{\text {de }}$ & $16.5 \pm 0.14^{\text {de }}$ & $10.50 \pm 0.29^{9}$ \\
\hline & n-hexane & $10.5 \pm 0.29^{9}$ & $12.96 \pm 0.02^{f}$ & $7.13 \pm 0.07^{h}$ \\
\hline & Ampiciline & $24.45 \pm 0.75^{a}$ & $17.75 \pm 0.14^{\mathrm{cd}}$ & $20 \pm 0.51^{c}$ \\
\hline \multirow[t]{6}{*}{ Aspergillus niger } & Methanol & $19.25 \pm 0.43^{c}$ & $14.60 \pm 0.23^{e}$ & $12.11 \pm 0.29^{f}$ \\
\hline & n-butanol & $21.13 \pm 0.51^{b}$ & $14.98 \pm 0.27^{e}$ & $19.50 \pm 0.29^{c}$ \\
\hline & Chloroform & $11.7 \pm 0.40^{f}$ & $17.15 \pm 0.66^{d}$ & $10.38 \pm 0.36^{9}$ \\
\hline & Ethyl acetate & $16.62 \pm 0.22^{d}$ & $16.38 \pm 0.22^{d}$ & $10.50 \pm 0.29^{9}$ \\
\hline & n-hexane & $10.25 \pm 0.14^{9}$ & $14.70 \pm 0.40^{e}$ & $7.63 \pm 0.36^{h}$ \\
\hline & Terbinafine & $24.38 \pm 0.79^{a}$ & $16.75 \pm 0.43^{d}$ & $20.25 \pm 0.43 b^{c}$ \\
\hline \multirow[t]{6}{*}{ Candida albicans } & Methanol & $18.75 \pm 0.72^{\text {de }}$ & $14.88 \pm 0.07^{h}$ & $12.15 \pm 0.37^{i}$ \\
\hline & n-butanol & $21.38 \pm 0.36^{b}$ & $15.23 \pm 0.13^{h}$ & $19.75 \pm 0.43^{c d}$ \\
\hline & Chloroform & $11.88 \pm 0.51^{i j}$ & $17.44 \pm 0.83^{e f}$ & $10.62 \pm 0.22^{\mathrm{jk}}$ \\
\hline & Ethyl acetate & $16.88 \pm 0.07^{\mathrm{fg}}$ & $16.13 \pm 0.36^{g h}$ & $10.38 \pm 0.36^{k}$ \\
\hline & n-hexane & $10.63 \pm 0.36^{\mathrm{jk}}$ & $14.95 \pm 0.55^{h}$ & $7.88 \pm 0.51^{1}$ \\
\hline & Terbinafine & $24.5 \pm 0.72^{\mathrm{a}}$ & $16.92 \pm 0.33^{f g}$ & $20.25 \pm 0.43^{b c}$ \\
\hline
\end{tabular}

Values are means \pm SD of three separate experiments $(p<0.05)$. Letters in superscript show the significance of the results against a single strain and among various parts.

methanolic extract give the following fractions: $n$-hexane, $9 \mathrm{~g}$; chloroform, $8 \mathrm{~g}$; ethyl acetate, $12 \mathrm{~g}: n$-butanol, 13 g. Leaves methanol extract was fractionated and $n$-hexane (12.4 g), chloroform (10 g), ethyl acetate $(8.3 \mathrm{~g})$ and $n$-butanol (10 g) fractions were obtained.

\section{Antibacterial and Antifungal assay}

Test microorganisms

Aspergillus niger ATCC 10595, Candida albicans ATCC 32612 were used as the fungal tested organisms and Pseudomonas aerugonisa locally isolated, Escherichia coli 
Table 4 Minimum inhibitory concentration $(\mathrm{mg} / \mathrm{ml})$ of $F$. benjamina against bacterial and fungal strains

\begin{tabular}{|c|c|c|c|c|}
\hline \multirow[t]{2}{*}{ Microbial strains } & \multirow{2}{*}{$\begin{array}{l}\text { Plant extracts } \\
\text { and fractions }\end{array}$} & \multicolumn{3}{|c|}{$\mathrm{MIC}$ as $\mathrm{mg} / \mathrm{ml}$} \\
\hline & & Stem & Root & Leaves \\
\hline \multirow[t]{6}{*}{ Bacillus Cerus } & Methanol & $1.03 \pm 0.03^{f}$ & $0.96 \pm 0.04^{f}$ & $1.02 \pm 0.02^{f}$ \\
\hline & n-butanol & $1.79 \pm 0.15^{\mathrm{bc}}$ & $1.49 \pm 0.02^{\text {de }}$ & $1.54 \pm 0.02^{\text {cde }}$ \\
\hline & Chloroform & $2.16 \pm 0.30^{\mathrm{a}}$ & $1.75 \pm 0.01^{\mathrm{bcd}}$ & $1.74 \pm 0.02^{\mathrm{bcd}}$ \\
\hline & Ethyl acetate & $1.78 \pm 0.15^{b c}$ & $1.92 \pm 0.03^{\mathrm{ab}}$ & $0.99 \pm 0.01^{f}$ \\
\hline & n-hexane & $1.41 \pm 0.15^{\mathrm{e}}$ & $1.54 \pm 0.03^{\text {cde }}$ & $1.59 \pm 0.01^{\text {cde }}$ \\
\hline & Ampiciline & $1.03 \pm 0.05^{f}$ & $0.5 \pm 0.01^{9}$ & $0.46 \pm 0.03^{9}$ \\
\hline \multirow[t]{6}{*}{ Pseudomonas aerugonisa } & Methanol & $2.11 \pm 0.10^{c d}$ & $2.01 \pm 0.01^{\text {cde }}$ & $0.54 \pm 0.02^{g h}$ \\
\hline & n-butanol & $0.83 \pm 0.06^{9}$ & $2.97 \pm 0.05^{\mathrm{a}}$ & $0.77 \pm 0.01^{g h}$ \\
\hline & Chloroform & $2.49 \pm 0.38^{b}$ & $1.75 \pm 0.03^{\text {ef }}$ & $0.86 \pm 0.03^{9}$ \\
\hline & Ethyl acetate & $2.18 \pm 0.19 b^{c}$ & $1.88 \pm 0.06^{\text {cde }}$ & $1.95 \pm 0.03^{\text {cde }}$ \\
\hline & n-hexane & $3.10 \pm 0.08^{\mathrm{a}}$ & $1.70 \pm 0.06^{\mathrm{ef}}$ & $1.55 \pm 0.03^{f}$ \\
\hline & Ampiciline & $1.85 \pm 0.06^{\mathrm{de}}$ & $0.48 \pm 0.01^{h}$ & $0.48 \pm 0.01^{h}$ \\
\hline \multirow[t]{6}{*}{ Escherichia coli } & Methanol & $1.94 \pm 0.07^{c}$ & $0.26 \pm 0.01^{i}$ & $0.28 \pm 0.02^{i}$ \\
\hline & $n$-butanol & $0.88 \pm 0.07^{d}$ & $0.76 \pm 0.01^{\text {def }}$ & $0.73 \pm 0.02^{\mathrm{d}-\mathrm{g}}$ \\
\hline & Chloroform & $3.16 \pm 0.16^{b}$ & $0.88 \pm 0.01^{d}$ & $0.47 \pm 0.02^{g h i}$ \\
\hline & 1Ethyl acetate & 冫 & $0.94 \pm 0.03^{d}$ & $0.52 \pm 0.02^{f-i}$ \\
\hline & n-hexane & $6.51 \pm 0.29^{a}$ & $0.81 \pm 0.01^{\text {de }}$ & $0.42 \pm 0.02^{h i}$ \\
\hline & Ampiciline & $0.34 \pm 0.06^{\mathrm{hi}}$ & $0.27 \pm 0.01^{i}$ & $0.26 \pm 0.01^{i}$ \\
\hline \multirow[t]{6}{*}{ Bacillus subtilis } & Methanol & $1.84 \pm 0.13^{b}$ & $0.58 \pm 0.04^{\text {hij }}$ & $0.55 \pm 0.02^{\text {hij }}$ \\
\hline & n-butanol & $0.65 \pm 0.06^{g h}$ & $0.78 \pm 0.02^{f g h}$ & $1.5 \pm 0.01^{\mathrm{cd}}$ \\
\hline & Chloroform & $1.25 \pm 0.31^{\mathrm{de}}$ & $3.44 \pm 0.05^{a}$ & $1.68 \pm 0.02^{b c}$ \\
\hline & Ethyl acetate & $0.95 \pm 0.04^{e f}$ & $1.92 \pm 0.07^{b}$ & $0.95 \pm 0.04^{\mathrm{efg}}$ \\
\hline & n-hexane & $3.42 \pm 0.30^{\mathrm{a}}$ & $3.18 \pm 0.09^{\mathrm{a}}$ & $1.61 \pm 0.02^{b c}$ \\
\hline & Ampiciline & $0.41 \pm 0.09^{i j}$ & $0.99 \pm 0.01^{\mathrm{ef}}$ & $0.28 \pm 0.0^{1 j}$ \\
\hline \multirow[t]{6}{*}{ Aspergillus niger } & Methanol & $0.52 \pm 0.006^{f}$ & $0.27 \pm 0.006^{i}$ & $0.52 \pm 0.02^{\mathrm{fg}}$ \\
\hline & n-butanol & $0.74 \pm 0.006^{\mathrm{e}}$ & $0.77 \pm 0.009^{\mathrm{de}}$ & $0.81 \pm 0.03^{c d}$ \\
\hline & Chloroform & $0.9 \pm 0.006^{c}$ & $0.84 \pm 0.023^{c}$ & $0.46 \pm 0.02^{h}$ \\
\hline & Ethyl acetate & $0.98 \pm 0.006^{a}$ & $0.54 \pm 0.021^{f}$ & $0.94 \pm 0.03^{a}$ \\
\hline & n-hexane & $0.83 \pm 0.006^{c}$ & $0.84 \pm 0.015^{c}$ & $0.77 \pm 0.01^{\text {de }}$ \\
\hline & Terbinafine & $0.49 \pm 0.006^{9}$ & $0.28 \pm 0.015^{i}$ & $0.27 \pm 0.02^{i}$ \\
\hline \multirow[t]{6}{*}{ Candida albicans } & Methanol & $0.52 \pm 0.009^{f}$ & $0.27 \pm 0.009^{i}$ & $0.51 \pm 0.023^{f}$ \\
\hline & n-butanol & $0.73 \pm 0.006^{e}$ & $0.77 \pm 0.012^{d}$ & $0.81 \pm 0.035^{c}$ \\
\hline & Chloroform & $0.89 \pm 0.019^{b}$ & $0.84 \pm 0.019^{c}$ & $0.45 \pm 0.009^{h}$ \\
\hline & Ethyl acetate & $0.97 \pm 0.006^{a}$ & $0.54 \pm 0.024^{f}$ & $0.94 \pm 0.020^{a}$ \\
\hline & $n$-hexane & $0.83 \pm 0.009^{c}$ & $0.84 \pm 0.019^{c}$ & $0.77 \pm 0.015^{d}$ \\
\hline & Terbinafine & $0.49 \pm 0.007^{9}$ & $0.27 \pm 0.018^{i}$ & $0.27 \pm 0.012^{i}$ \\
\hline
\end{tabular}

Values are means \pm SD of three separate experiments $(p<0.05)$. Letters in superscript show the significance of the results against a single strain and among various parts. Ampiciline and Terbinafine were used as standards for bacteria and fungi, respectively.

ATCC 25922, Bacillus subtilis JS 2004, Bacillus cerus locally isolated were used as the bacterial tested organisms. The pure bacterial and fungal strains were obtained from the Department of Veterinary Microbiology, University of Agriculture, Faisalabad, Pakistan. The bacterial strains were cultured overnight at $37^{\circ} \mathrm{C}$ in nutrient agar (Oxoid,
Hampshire, UK) while fungal strains were cultured overnight at $28^{\circ} \mathrm{C}$ using potato dextrose agar (Oxoid).

Antibacterial and antifungal assay by disc diffusion method Antimicrobial activity was determined by using the disc diffusion method [21]. All samples (dry residue) were 
Table 5 HPLC analysis of stem, root and leaves for phenolic acids

\begin{tabular}{llll}
\hline Phenolic acids & Stem $(\mathbf{p p m})$ & Root $(\mathbf{p p m})$ & Leaves $(\mathbf{p p m})$ \\
\hline Chlorogenic acid & 0.936 & 5.538 & $\mathrm{ND}$ \\
Paracoumaric acid & 0.11 & 0.13 & $\mathrm{ND}$ \\
Ferulic acid & 2.88 & 1.29 & $\mathrm{ND}$ \\
Syringic acid & $\mathrm{ND}$ & 0.54 & $\mathrm{ND}$ \\
Caffeic acid & $\mathrm{ND}$ & $\mathrm{ND}$ & 47.61 \\
\hline *ND $=$ not detected & & &
\end{tabular}

dissolved in $10 \%$ sterile dimethyl sulfoxide. The discs (6 $\mathrm{mm}$ diameter) were impregnated with $10 \mathrm{mg} / \mathrm{mL}$ extract/fractions $(100 \mu \mathrm{L} /$ disc) placed aseptically on the inoculated agar. Discs injected with $100 \mu \mathrm{L}$ of respective solvents served as a negative controls, rifampcin $(100 \mu \mathrm{L} /$ disc) (Oxoid) and fluconazole (100 $\mu \mathrm{L} / \mathrm{disc}$ ) (Oxoid) were used as positive reference for bacteria and fungi, respectively. The Petri dishes were incubated at $37 \pm 0.1^{\circ} \mathrm{C}$ for $20-24 \mathrm{~h}$ and $28 \pm 0.3^{\circ} \mathrm{C}$ for $40-48 \mathrm{~h}$ for bacteria and fungi, respectively. At the end of period, the inhibition zones formed on the media were measured. The positive antimicrobial activity was read based on growth inhibition zone.

\section{Minimum inhibitory concentrations (MIC) of plant extracts} Minimum inhibitory concentration both for bacterial and fungal strains was measured as reported in literature [31].

\section{Determination of total phenolic contents}

Amount of total phenolic contents were assessed using Folin-Ciocalteu reagent procedure [32]. Briefly, $1 \mathrm{mg}$ of dry mass of crude extracts/fractions was mixed with $0.5 \mathrm{~mL}$ of Folin-Ciocalteu reagent and $7.5 \mathrm{~mL}$ deionized water. The mixture was kept at room temperature for $10 \mathrm{~min}$ and then $1.5 \mathrm{~mL}$ of $20 \%$ sodium carbonate (w/v) was added. The mixture was heated in a water bath at $40^{\circ} \mathrm{C}$ for $20 \mathrm{~min}$ and then cooled in an ice bath; finally absorbance at $755 \mathrm{~nm}$ was measured (Hitachi U-2001 spectrophotometer). Amounts of TP were calculated using a calibration curve for gallic acid (10-100 ppm)

Table 6 Percent haemolysis caused by $F$. benjamina

\begin{tabular}{llll}
\hline Plant extract and fraction & Stem & Root & Leaves \\
\hline Methanol & $1.54 \pm 0.023$ & $1.89 \pm 0.061$ & $1.93 \pm 0.241$ \\
n-butanol & $2.49 \pm 0.061$ & $1.64 \pm 0.040$ & $1.13 \pm 0.023$ \\
Chloroform & $3.36 \pm 0.080$ & $1.46 \pm 0.061$ & $3.29 \pm 0.061$ \\
Ethyl acetate & $2.26 \pm 0.061$ & $1.81 \pm 0.100$ & $2.09 \pm 0.061$ \\
$n$-hexane & $2.91 \pm 0.046$ & $0.90 \pm 0.100$ & $1.92 \pm 0.105$ \\
Triton & $99.8 \pm 0.612$ & $99.8 \pm 0.612$ & $99.8 \pm 0.612$ \\
Phosphate buffer saline & 0 & 0 & 0 \\
\hline
\end{tabular}

Values are means \pm SD of three separate experiments $(p<0.05)$.
Table 7 Chemical composition of $F$. benjamina (Stem, Root) essential oils

\begin{tabular}{llll}
\hline $\begin{array}{l}\text { Retention } \\
\text { time }(\boldsymbol{m i n})\end{array}$ & Chemical constituents & \multicolumn{2}{c}{$\%$ Area } \\
\cline { 3 - 4 } & & Stem & Root \\
\hline 2.17 & 2-Pentanone & 0.1 & n.d \\
10.26 & Hexadecanoic acid & 0.34 & 1.98 \\
11.04 & Palmitic acid & 0.84 & 1.89 \\
11.91 & $9,12-$-Octadecadienoic acid & 0.47 & 3.55 \\
0.16 & Methanamine & n.d & 1.62 \\
2.297 & Cyclopentanone & n.d & 0.12 \\
10.63 & Methyl-2 Phenylindole & n.d & 0.12 \\
12.53 & Cyclopropaneoctanal & n.d & 0.44 \\
15.73 & Arsenous acid & n.d & 0.15 \\
\hline
\end{tabular}

$(\mathrm{R} 2=0.9986)$. The results were expressed as gallic acid equivalents (GAE) of dry plant matter.

\section{Determination of total flavonoid contents}

The total flavonoid content in plant extract and fractions was determined by following the already reported procedure [32]. Plant extract/fractions of each material ( $1 \mathrm{~mL}$ containing $0.1 \mathrm{~g} / \mathrm{mL}$ ) was placed in a $10 \mathrm{~mL}$ volumetric flask, then added distilled water $5 \mathrm{~mL}$ and $0.3 \mathrm{~mL}$ of $5 \% \mathrm{NaNO}_{2}$ was added to each volumetric flask initially; after $5 \mathrm{~min}, 0.6 \mathrm{~mL}$ of $10 \% \mathrm{AlCl}_{3}$ was added. After another $5 \mathrm{~min}, 2 \mathrm{~mL}$ of $1 \mathrm{M} \mathrm{NaOH}$ was added and volume made up with distilled water. Then solution was mixed. At $510 \mathrm{~nm}$ absorbance of the reaction mixture was taken using a spectrophotometer. Total flavonoid content were evaluated as catechin equivalents (CE g/100 g of dry plant matter).

\section{Antioxidant analysis}

\section{Measurement of reducing potential}

The reducing potential of plant extract was determined by methods reported in literature [33].

Equivalent volume of plant crude extracts/fractions containing $2.5-10 \mathrm{mg}$ of dry matter was mixed with sodium phosphate buffer $(5.0 \mathrm{~mL}, 0.2 \mathrm{M}, \mathrm{pH} 6.6)$ and potassium ferricyanide $(5.0 \mathrm{~mL}, 1.0 \%)$; the mixture was incubated at $50^{\circ} \mathrm{C}$ for $20 \mathrm{~min}$. Then $5 \mathrm{~mL}$ of $10 \%$ trichloroacetic acid was added and centrifuged at $980 \mathrm{xg}$ for $10 \mathrm{~min}$ at $5^{\circ} \mathrm{C}$ in a refrigerated centrifuge. The upper layer of the solution $(5.0 \mathrm{~mL})$ was diluted with $5.0 \mathrm{~mL}$ of distilled water and ferric chloride $(1.0 \mathrm{~mL}, 0.1 \%)$ and absorbance noted at $700 \mathrm{~nm}$ (Hitachi U-2001 spectrophotometer). The measurement was run in triplicate and results averaged.

\section{DPPH free radical scavenging assay}

The 2,2-diphenyl-1-picrylhydrazyl radical (DPPH) assay was carried out spectrophotometrically as described by Bozin and coworkers [34]. Aliquots $(50 \mu \mathrm{L})$ of various 
concentrations $(10-100 \mu \mathrm{g} / \mathrm{mL})$ of the plant extract/fractions was added to $5 \mathrm{~mL}$ of a $0.004 \%$ methanolic solution of DPPH. After $0.5 \mathrm{~h}$ incubation period at room temperature, the absorbance was read against a blank at $517 \mathrm{~nm}$ :

$$
\text { Inhibition }(\%)=100 \times(A \text { blank }-A \text { sample } / A \text { blank })
$$

where Ablank is the absorbance of the control reaction (containing all reagents except the test compound) and Asample is the absorbance of the test compound. Extract/fraction concentration providing 50\% inhibition (IC50) was calculated from a graph plotting percentage inhibition against extract concentration.

\section{Antioxidant activity determination in linoleic acid system}

The antioxidant activity was also determined in terms of measurement of percent inhibition of peroxidation in linoleic acid system, as previously reported [35]. Extracts/frations (5 mg) were added to a solution mixture of linoleic acid $(0.13 \mathrm{~mL}), 99.8 \%$ ethanol $(10 \mathrm{~mL})$ and $10 \mathrm{~mL}$ of $0.2 \mathrm{M}$ sodium phosphate buffer $(\mathrm{pH}=7.4)$. Total mixture was diluted to $25 \mathrm{~mL}$ with distilled water. The solution was incubated at $40^{\circ} \mathrm{C}$ and the degree of oxidation was measured following thiocyanate method with $10 \mathrm{~mL}$ of ethanol (75\%), $0.2 \mathrm{~mL}$ of an aqueous solution of ammonium thiocyanate (30\%), $0.2 \mathrm{~mL}$ of sample solution and $0.2 \mathrm{~mL}$ of ferrous chloride $(\mathrm{FeCl} 2)$ solution (20 $\mathrm{mM}$ in $3.5 \% \mathrm{HCl}$ ) being added sequentially. After 3 min of stirring, the absorption values of mixtures measured at $500 \mathrm{~nm}$ were taken as peroxide contents. A control was performed with linoleic acid but without extracts. Synthetic antioxidants; butylated hydroxytoluene (BHT) was used as positive control. The maximum peroxidation level observed as $360 \mathrm{~h}$ (15 days) in the sample that contained no antioxidant component was used as a test point.

Percent inhibition of linoleic acid peroxidation $=100-$ [(Abs. increase of sample at $360 \mathrm{~h} / \mathrm{abs}$. increase of control at $360 \mathrm{~h}$ ) x100], was calculated to express antioxidant activity.

\section{Hemolytic activity}

Hemolytic activity was checked by following the reported method [29]. Briefly, three $\mathrm{ml}$ of freshly obtained heparinzed human blood was gently mixed, poured into sterile $15 \mathrm{ml}$ falcon tube and centrifuged for $5 \mathrm{~min}$ at $850 \times \mathrm{g}$. The supernatant was poured off and viscous pellet washed three additional times with $5 \mathrm{ml}$ of chilled $\left(4^{\circ} \mathrm{C}\right)$ sterile isotonic phosphate-buffered saline solution (PBS) solution, adjusted to $\mathrm{pH}=7.4$. The washed cells were suspended in the $20 \mathrm{ml}$ chilled, saline PBS buffer. Erythrocytes were counted on a haemacytometer (Fisher ultra plane Neubauer ruling). Twenty $\mu \mathrm{l}$ of plant extract $(15 \mathrm{mg} / \mathrm{ml})$ in five different solvent was taken in $20 \mathrm{ml}$ Eppendorff tubes. For each assay, $0.1 \%$ Triton $\mathrm{X}-100$ was taken as a positive control, $100 \%$ blood lysis and Phosphate buffer saline (PBS) was taken for each assay as a negative control (0\% lysis). To each $2 \mathrm{ml}$ Eppendorff tube already containing $20 \mu \mathrm{l}$ sample, $180 \mu \mathrm{l}$ diluted blood cell suspension was added and mixed. Tubes were incubated for $35 \mathrm{~min}$ at $37^{\circ} \mathrm{C}$ and agitate after $10 \mathrm{~min}$. Immediately after incubation, the tubes were placed on ice for $5 \mathrm{~min}$ and then centrifuged for $5 \mathrm{~min}$ at $1310 \mathrm{xg}$. After centrifugation, $100 \mu \mathrm{l}$ supernatant was taken from the tubes and diluted with $90 \mu$ chilled PBS. Then, $200 \mu$ l were placed into 96 well plates. After this, absorbance at $576 \mathrm{~nm}$ was measured. Triton-X (0.1\%) was taken as a positive control for $100 \%$ lyses and PBS buffer as negative control for $0 \%$ lyses. The experiment was done in triplicate and mean \pm S.E. was calculated using the following formula:

$$
\text { percent hemolysis }=(\mathrm{Hb} \mathrm{ABS} / \mathrm{Hb} 100) \times 100
$$

\section{Sample extraction for HPLC analysis}

The methanolic extracts of stem root and leaves were prepared by using $0.5 \mathrm{~g}$ of dry-ground sample and $20 \mathrm{~mL}$ of solvent. The mixtures were shaken in a c24k refrigerated incubator shaker (NJ, USA) at room temperature for $60 \mathrm{~min}$. Then, the mixtures were centrifuged in a Universal 320r Hettich Zenrifugen (Tuttlingen, Germany) at $9000 \mathrm{rpm}$ for $5 \mathrm{~min}$. at $4^{\circ} \mathrm{C}$. The supernatant was recovered and used for the determination of phenolic acids by HPLC [36]. The supernatants were filtered through a $45 \mu \mathrm{m}$ filter prior to analysis. All the extractions were performed in duplicate and the supernatants were kept at $-20^{\circ} \mathrm{C}$ until further analysis.

An HPLC (model LC-10A, Shimadzu, Japan) equipped with two LC-10 AS pumps, SCL-10A system control unit, Rheodyne injector, CTO-10A column oven, SPD10A UV-vis detector and data acquisition class CSW32 soft ware was used.

\section{Gas Chromatography/Mass Spectrometry analysis}

The GC-MS analysis of the essential oils was carried out using a GC 6850 Network gas chromatographic system, equipped with a 7683 B Series auto injector and a 5973 I inert mass selective detector (Agilent Technologies USA), using a HP-5 MS capillary column having $5 \%$ phenyl polysiloxane as a stationary phase, column length $30.0 \mathrm{~m}$, i.d. $0.25 \mathrm{~mm}$ and film thickness $0.25 \mu \mathrm{m}$. One $\mu \mathrm{l}$ of sample was injected in the split mode with split ratio 30:1 at a temperature of $300^{\circ} \mathrm{C}$. Helium was used as carrier gas with a constant flow rate of $1.5 \mathrm{ml} / \mathrm{min}$. The temperature program was as follows: initial temperature $150^{\circ} \mathrm{C}$, held for $1 \mathrm{~min}$ and then ramping at rate of $10^{\circ} \mathrm{C} / \mathrm{min}$ up to $290^{\circ} \mathrm{C}$, kept constant for $5 \mathrm{~min}$. The temperature of transfer line was $300^{\circ} \mathrm{C}$. Electron ionization mode with the ionization 
energy of $70 \mathrm{eV}$ having mass range scanned $3-500 \mathrm{~m} / \mathrm{z}$ was used for mass spectra determination. The temperature of ion source was $230^{\circ} \mathrm{C}$ and that of MS quadropole $150^{\circ} \mathrm{C}$. The identification of components was based on comparison of their mass spectra with those of NIST mass spectra library $[37,38]$.

\section{Statistical analysis}

Two factor completely randomized design (CRD) was applied and significant difference among means was worked out using Duncan's multiple range (DMR) test at $5 \%$ level of significance.

\section{Conclusion}

Present study ascertained the potency of the F. benjamina as a potential source of natural antioxidants and antimicrobial agents. The root and leaves showed good antioxidant activity, whereas stem extract and fractions revealed good antimicrobial activity. Ficus benjamina disclosed substantial bioactivity, being root extract and fractions the most active. This plant can be regarded as a potential source of antioxidant and antimicrobial agents.

\section{Competing interests}

The authors declare that they have no competing interests.

\section{Authors' contributions}

MI, NR, KR, MZ, UAR, AN, and MR made a significant contribution to experiment design, acquisition of data, analysis and drafting of the manuscript. MZUH and HZEJ have made a substantial contribution to interpretation of data, drafting and carefully revising the manuscript for intellectual content. All authors read and approved the final manuscript.

\section{Acknowledgments}

The authors would like to extend their sincere appreciation to Scientific Research (scientific instruments) at King Saud University for funding through the Research Group Project no RGP-VPP-236.

\section{Author details}

'Department of Chemistry, Government College University Faisalabad, Faisalabad 38000, Pakistan. ${ }^{2}$ The Patent Office, Karachi, Pakistan. ${ }^{3}$ Deanship of Scientific Research, College of Engineering, King Saud University, Riyadh 11421, Saudi Arabia. ${ }^{4}$ Department of Chemistry, College of Science, King Saud University, Riyadh 1145, Saudi Arabia. ${ }^{5}$ Department of Crop Science, Faculty of Agriculture, 43400 UPM Serdang, Selangor, Malaysia. ${ }^{6}$ University Community Transformation Centre, Industry-Community Engagement, 43400 UPM Serdang, Selangor, Malaysia.

Received: 26 November 2013 Accepted: 28 January 2014 Published: 13 February 2014

\section{References}

1. Riffle RL: The Tropical Look. Portland, Oregon: Timber Press, Inc; 1998

2. Hawaiian Ecosystems at Risk project (HEAR): Invasive plant species: Ficus benghalensis L., Moraceae. Pacific Island Ecosystems at Risk; 2001. Available: http://www.hear.org/species/ficus_benghalensis/ (Accessed: January 16, 2002).

3. Parajuli SP: Ethnobotanical studies of at khandbari muncipilty of Sankhuwasabha. Banko Janak 2000, 10:29-34.

4. Kanaujia VK, Rirchhaiya HK, Kailasiya SD, Verma M, Yadav RD, Shivhare D: Evaluation of hepatoprotective activity on the leaves of Ficus benjamina Linn. J Nat Prod Plant 2011, 1:59-69.

5. Sirisha N, Sreenivasulu M, Sangeeta K, Chetty CM: Antioxidant properties of Ficus species, a review. Int J Pharma Techn Res 2010, 4:2174-2182.
6. Almahyl HA, Rahmani M, Sukarp MA, Ali AA: Investigation on the chemical constituents of the leaves of Ficus elastica Roxb. and their antimicrobial activity. Pertanika J Sci Tech 2003, 11:57-63.

7. Ogunwande IA, Jimoh R, Ajetunmobi AA, Avoseh NO, Flamini G: Essential oil composition of Ficus benjamina (Moraceae) and Irvingia barteri (Irvingiaceae). Nat Prod Commun 2012, 7:1673-1675.

8. Yarmolinsky L, Zaccai M, Ben-Shabat S, Mills D, Huleihel M: Antiviral activity of ethanol extracts of Ficus benjamina and Lilium candidum in vitro. New Biotech 2009, 26:307-313.

9. Dai J, Shen D, Yoshida WY, Parrish SM, Williams PG: Isoflavonoids from Ficus benjamina and their inhibitory activity on BACE1. Planta Med 2012, 78:1357-1362.

10. Yarmolinsky L, Huleihel M, Zaccai M, Ben-Shabat S: Potent antiviral flavone glycosides from Ficus benjamina leaves. Fitoterapia 2012, 83:362-367.

11. Zia-Ul-Haq M, Ahmad S, Shad MA, lqbal S, Qayum M, Ahmad A, Luthria DL, Amarowicz R: Compositional studies of some of lentil cultivars commonly consumed in Pakistan. Pak J Bot 2011, 43:1563-1567.

12. Zia-Ul-Haq M, Amarowicz R, Ahmad S, Riaz M: Antioxidant potential of some pea (Pisum sativum L.) cultivars commonly consumed in Pakistan. Oxid Communication 2013, 36:1046-1057.

13. Zia-Ul-Haq M, Ćavar S, Qayum M, Imran I, De Feo V: Compositional studies: antioxidant and antidiabetic activities of Capparis decidua (Forsk.) Edgew. Int J Mol Sci 2011, 12:8846-8861.

14. Zia-Ul-Haq M, Ahmad S, labal S, Luthria DL, Amarowicz R: Antioxidant potential of lentil cultivars commonly consumed in Pakistan. Oxid Comm 2011, 34:819-831.

15. Zia-Ul-Haq M, Ahmad S, Amarowicz R, Defeo V: Antioxidant activity of the extracts of some cowpea (Vigna unguiculata (L) Walp.) cultivars commonly consumed in Pakistan. Molecules 2013, 18:2005-2017.

16. Zia-Ul-Haq M, Ahmad S, Qayum M, Ercişli S: Compositional studies and antioxidant potential of Albizia lebbeck (L.) Benth. Turk J Bio 2013, 37:25-32.

17. Zia-Ul-Haq M, Riaz M, De Feo V: Ipomea hederacea Jacq.: a medicinal herb with promising health benefits. Molecules 2012, 17:13132-13145.

18. Zia-Ul-Haq M, Shah MR, Qayum M, Ercisli S: Biological screening of selected flora of Pakistan. Bio Res J 2012, 45:375-379.

19. Zia-UI-Haq M, Ahmad S, Calani L, Mazzeo T, Rio DD, Pellegrini N, De Feo V: Compositional study and antioxidant potential of Ipomoea hederacea Jacq. and Lepidium sativum L. seeds. Molecules 2012, 17:10306-10321.

20. Zia-Ul-Haq M, Stanković M, Rizwan K, Defeo V: Grewia asiatica L., a food plant with multiple uses. Molecules 2013, 18:2663-2682.

21. Rizwan K, Zubair M, Rasool N, Riaz M, Zia-Ul-Haq M, DeFeo V: Phytochemical and biological studies of Agave attenuata. Int J Mo/ Sci 2012, 13:6440-6451.

22. Abdel-hameed ESS: Total phenolic contents and free radical scavenging activity of certain Egyptian Ficus species leaf samples. Food Chem 2009, $14: 1271-1277$

23. Adebayo EA, Ishola OR, Taiwo OS, Majolagbe ON, Adekeye BT: Evaluations of the methanol extract of Ficus exasperate stem, bark, leaf and root for phytochemical analysis and antimicrobial activities. Afri J Plant Sci 2009, 3:283-287.

24. Karimi E, Oskoueian E, Hendra R, Jaafar HZE: Evaluation of Crocus sativus L. Stigma Antioxidant Activity and Its Phenolic and Flavonoid Compounds. Molecules 2010, 15(9):2644.

25. Karimi E, Jaafar HZE, Ahmad S: Phytochemical analysis and antimicrobial activities of methanolic extracts of leaf, stem and root from different varieties of Labisa pumila Benth. Molecules 2011, 16:4438-4450.

26. Jaafar HZE, Ibrahim MH, Karimi E: Phenolics and Flavonoids Compounds, Phenylanine Ammonia Lyase and Antioxidant Activity Responses to Elevated CO2 in Labisia pumila (Myrisinaceae). Molecules 2012, 17(6):6331-6347

27. Proestos C, Chorianopoulos N, Nychas GJE, Komaitis M: RP-HPLC analysis of the phenolic compounds of plant extracts. Investigation of their antioxidant capacity and antimicrobial activity. J Agric Food Chem 2005, 53:1190-1195

28. Merkl R, Hradkova I, Filip V, Smidrkal J: Antimicrobial and antioxidant properties of phenolic acids alkyl esters. Czech J Food Sci 2010, 28:275-279.21

29. Powell WA, Catranis CM, Maynard CA: Design of self-processing antimicrobial peptides for plant protection. Lett Appli Micr 2000, 31:163-168.

30. Sharma P, Sharma JD: In vitro hemolysis of human erythrocytes by plant extracts with antiplasmodial activity. J Ethnopharmacol 2001, 74:239-243 
31. Sarker SD, Nahar L, Kumarasamy Y: Microtitre plate-based antibacterial assay incorporating resazurin as an indicator of cell growth, and its application in the in vitro antibacterial screening of phytochemicals. Methods 2007, 42:321-324.

32. Zubair M, Hassan S, Rizwan K, Rasool N, Riaz M, Zia-Ul-Haq M, Defeo V: Antioxidant potential and oil composition of Callistemon viminalis leaves. The Sci World J 2013, 2013:1-8.

33. Yen GC, Duh PD, Chuang DY: Antioxidant activity of anthraquinones and anthrone. Food Chem 2007, 70:307-315.

34. Bozin B, Mimica-dukie N, Simin N, Anackov G: Characterization of the volatile composition of essential oil of some lamiaceae species and the antimicrobial and antioxidant activities of the entire oils. J Agri Food Chem 2006, 54:1822-1828.

35. Iqbal S, Bhanger MI: Antioxidant properties and components of some commercially available varieties of rice bran in Pakistan. Food Chem 2005, 93:265-272.

36. Demiray S, Pintado ME, Castro PML: Evaluation of phenolic profiles and antioxidant activities of Turkish medicinal plants: Tilia argentea, Crataegi folium leaves and Polygonum bistorta roots. World Acad Sci Eng Tech 2009, 54:312-317.

37. Massada Y: Analysis of essential oils by gas chromatography and mass spectrometry. New York: Wiley; 1976.

38. Mass Spectral Library, NIST/EPA/NIH: USA; 2002. http://www.nist.gov/srd/ nistla.cfm.

doi:10.1186/1752-153X-8-12

Cite this article as: Imran et al:: Chemical composition and Biological studies of Ficus benjamina. Chemistry Central Journal 2014 8:12.

\section{Publish with ChemistryCentral and every scientist can read your work free of charge \\ "Open access provides opportunities to our colleagues in other parts of the globe, by allowing anyone to view the content free of charge." \\ W. Jeffery Hurst, The Hershey Company. \\ - available free of charge to the entire scientific community \\ - peer reviewed and published immediately upon acceptance \\ - cited in PubMed and archived on PubMed Central \\ - yours - you keep the copyright \\ Submit your manuscript here: \\ http://www.chemistrycentral.com/manuscript/<smiles>c1ccccc1</smiles> \\ Chemistry Central}

